\title{
Community-Acquired Acute Kidney Injury Induced By Drugs In Older Patients: A Multifactorial Event
}

This article was published in the following Dove Press journal:

Clinical Interventions in Aging

\author{
Laurine Robert' \\ Grégoire Ficheur ${ }^{2}$ \\ Sophie Gautier ${ }^{3}$ \\ Alexandre Servais' \\ Michel Luyckx ${ }^{4}$ \\ Julien Soula $\mathbb{D}^{\prime}$ \\ Bertrand Decaudin ${ }^{5}$ \\ François Glowacki (iD ${ }^{6}$ \\ François Puisieux' \\ Emmanuel Chazard (iD ${ }^{2}$ \\ Jean-Baptiste Beuscart' \\ 'Univ. Lille, CHU Lille, EA2694, Lille, \\ F-59000, France; ${ }^{2}$ Univ. Lille, CHU Lille, \\ EA2694, Public Health Department, Lille, \\ F-59000, France; ${ }^{3}$ Univ. Lille, CHU Lille, \\ UMR II7I, Department of \\ Pharmacology, Lille, F-59000, France; \\ ${ }^{4}$ Univ. Lille, EA7365, F-59000 Lille, CH \\ Denain, Department of Pharmacy, \\ Denain, F-59220, France; ${ }^{5}$ Univ. Lille, \\ CHU Lille, EA7365, Department of \\ Pharmacy, Lille, F-59000, France; ${ }^{6}$ Univ. \\ Lille, CHU Lille, EA4483 IMPECS, \\ Department of Nephrology, Lille, F- \\ 59000, France
}

Purpose: Community-acquired acute kidney injury (CA-AKI) is a frequent and severe adverse drug reaction (ADR) among older patients. The combination of drugs and other CA-AKI risk factors was barely evaluated. The objectives of our study were to both accurately identify CA-AKI induced by drugs in older patients, and to describe their combination with other risk factors.

Patients and methods: We conducted a retrospective, single-center study in a general hospital over a two-year period. An automated detection identified CA-AKI according to KDIGO criteria, amongst 4,767 eligible inpatient stays among patients aged 75 years or older. Two independent experts reviewed all CA-AKI events to adjudicate drug involvement (Naranjo scale), identify inappropriate prescriptions (STOPP criteria), evaluate avoidability (Hallas criteria) and identify combined risk factors.

Results: An expert review confirmed 713 CA-AKI (15.0\% of inpatient stays) and determined that 419 (58.8\%) CA-AKI were induced by drugs. A multifactorial cause (i.e., at least one drug with a precipitating factor) was found in $63.2 \%$ of drug-induced CA-AKI. Most of the drug-induced events were avoidable $(66.8 \%)$, mainly in relation to a multifactorial cause. Conclusion: Drug-induced CA-AKI were frequent, multifactorial events in hospitalized older patients and their prevention should focus on combinations with precipitating factors. Keywords: adverse drug reactions, elderly, prevention, acute kidney injury

\section{Introduction}

Acute kidney injury (AKI) is a frequent and severe disease associated with an increased risk of hospitalization or death. ${ }^{1-5}$ Older patients are at a higher risk of AKI than younger patients ${ }^{6-9}$ due to an increased prevalence of chronic kidney disease, cardiovascular morbidities, and polypharmacy. Indeed, AKI is often considered as an adverse drug reaction (ADR), ${ }^{10,11}$ with drug involvement present in the occurrence of $20.0 \%$ to $66.0 \%$ of AKI. ${ }^{12,13}$

AKI can occur both in primary care (i.e., community-acquired AKI (CA-AKI)) and in hospital settings (i.e., hospital-acquired AKI (HA-AKI)). CA-AKI account for $54.5 \%$ to $79.4 \%$ of AKI cases. ${ }^{14-17}$ Detection and prevention of CA-AKI are important because the mortality rates associated with CA-AKI measure $25 \%$ at 30 days and $45 \%$ at 3 years. ${ }^{3,4}$ Prevention mainly focusses on improving the prescription of drugs and strengthening their monitoring ${ }^{12}$ as $59.9 \%$ to $72.0 \%$ of CA-AKI are induced by drugs. ${ }^{16,18}$

Drugs are not the only risk factors for AKI and many precipitating factors can contribute to the occurrence of AKI. ${ }^{2,6,19}$ However, risk factors for AKI were most often independently evaluated through multivariate analysis. ${ }^{20-22}$ This approach cannot describe combination of drugs and other precipitating factors of AKI. A
Correspondence: Laurine Robert CERIM - EA 2694, Faculté de Médecine Pôle Recherche, I Place de Verdun, Lille Cedex 59045 France

Email laurine.robert.etu@univ-lille.fr 
study performed in England and Scotland showed a cumulative effect of AKI risk factors. ${ }^{19}$ These results have an important impact on ADR prevention strategies. However, an accurate analysis of the drugs involved in the occurrence of CA-AKI with validated tools and their relations with other risk factors was not performed.

The objective of our study was to accurately identify CA-AKI induced by drugs among older patients, and to describe their combination with other risk factors in order to assess the avoidability of CA-AKI.

\section{Methods}

\section{Study Design}

This retrospective, observational, single-center cohort study was performed in a general hospital in northern France. The hospital comprehends surgery, pulmonology, cardiology, angiology, hepato-gastro-enterology, internal medicine and emergency departments. Our analysis encompassed all stays in the general hospital recorded between January 1, 2012 and December 31, 2013 (29,970 in total). All extracted study data were anonymous.

Our study was performed from the perspective of the health professionals, i.e. when faced with an individual patient. In this context, causality assessment tools are recommended for the validation of individual cases to identify combined risk factors for CA-AKI. The study did not aim to conduct pharmacoepidemiological or epidemiological studies of CA-AKI (e.g., mortality rate, length of inpatient stay, and Charlson comorbidity index were not estimated).

\section{Ethics Approval}

The study was registered with the French National Data Protection Commission (Commission Nationale de l'Informatique et des Libertés; reference numbers 1487204 and VIa0335797v) and was approved by the local investigational review board (Comité de Protection des Personnes Nord-Ouest IV) in January 2008.

\section{Data Collection}

For each inpatient stay, the following items of information were extracted from the linked electronic medical records: administrative data (age, gender, etc.), the diagnostic code associated with the inpatient stay (according to the International Classification of Disease 10th Revision $(\mathrm{ICD}-10))^{23}$ medications administered during the stay (according to the Anatomical Therapeutic Chemical (ATC) terminology) $^{24}$ laboratory results (according to the Committee on Nomenclature, Properties and Units (C-NPU) terminology ${ }^{25}$ anonymized discharge letters, and free-text hospital care reports. ${ }^{26}$

\section{Inclusion And Exclusion Criteria}

We intended to include all CA-AKI events in patients aged 75 and over. AKI was defined according to the Kidney Disease Improving Global Guidelines (KDIGO) classification, i.e. an increase in serum creatinine by at least $0.3 \mathrm{mg} / \mathrm{dl}$ within $48 \mathrm{hrs}$ (if baseline serum creatinine $<1.2 \mathrm{mg} / \mathrm{dL}$ ) or increase in serum creatinine to at least 1.5 times baseline within the prior 7 days (Table S1). AKI was initially defined as CA-AKI if the increase in serum creatinine was observed during the first $48 \mathrm{hrs}$ of hospitalization. ${ }^{3,15}$ It was deemed that this approach may not detect AKI originating in a community setting, with patients having a high serum level of creatinine at the time of hospital admission. AKI was therefore secondly defined as CA-AKI if a decrease of serum creatinine was observed during the inpatient stay, and if the serum creatinine value at admission time was higher than the lowest value during inpatient stay. AKI was defined by either a $50 \%$ decrease between two creatinine value within 7 days, either a difference of $0.3 \mathrm{mg} / \mathrm{dl}$ within 48 hrs (if lowest serum creatinine $<1.2 \mathrm{mg} / \mathrm{dL}$ ). Examples of possible cases are provided in the Supporting Information (Figure S1).

Eligibility for the study required a minimum stay duration of 2 days and at least 2 creatinine values (including one at the time of admission). Only inpatients of 75 years and over, fitting these criteria were eligible for the study. All AKI events were automatically detected according to these criteria and then reviewed by two independent experts (LR, AS). AKI events were secondarily excluded in case of: 1) erroneous-automated detection; 2) missing data (in relation to the drugs prescribed, the diagnosis or the laboratory results). Cohen's Kappa evaluated the extent of agreement between the two experts for the validation of CA-AKI events. In fact, Cohen's Kappa was 0.87 , indicating near perfect agreement (defined by convention as a value between 0.81 and 0.99). Any disagreement was resolved by discussion and consensus. A third reviewer (JBB) arbitrated as necessary.

\section{Expert Review Of Each AKI Event}

All detected AKI events were analyzed by two independent reviewers (LR, AS) using the ADE-Scorecards ${ }^{\circledR}$ 
software. This latter tool was developed as part of the European Patient Safety through Intelligent Procedure (PSIP) project. The ADE-Scorecards ${ }^{\circledR}$ interface summarized the data available for each stay (administrative data, lab results, diagnostic data, drug prescriptions and freetext documents). ${ }^{27,28}$ This tool was used for data extraction, as detailed below. Only well-known risk factors for AKI were extracted, as the study did not intend to find new risk factors. Obstructive AKI was excluded from the drug analysis because the reviewers considered that drug involvement was secondary in the occurrence of the AKI, even if the Naranjo score was $\geq 1$. The reviewers then evaluated the relationship between drugs and AKI, inappropriateness of the prescription, avoidability of the event. Any disagreement was resolved by discussion and consensus. A third reviewer (JBB) arbitrated as necessary.

\section{Predisposing And Precipitating Factors}

Chronic kidney disease, high blood pressure, chronic heart failure and diabetes were considered as predisposing factors of $\mathrm{AKI}^{2,6,15,16,19}$ and were identified either in the discharge letters or in the ICD-10 coding. Chronic kidney disease also had to be defined by an estimated glomerular filtration rate $(\mathrm{eGFR})<60 \mathrm{ml} / \mathrm{min}$ according to CKDEPI. ${ }^{29,30}$ Infection, acute heart failure, dehydration, surgery, rhabdomyolysis, ascites caused by cirrhosis and administration of contrast agents were considered as precipitating factors of AKI and were identified either in the discharge letters or in the ICD-10 coding. ${ }^{2,6,15,16,19}$ Precipitating factors had to be present at admission (i.e., during the first $48 \mathrm{hrs}$ of hospitalization) and before the occurrence of the AKI.

\section{Drugs}

Drugs known for their potential involvement in AKI were considered: angiotensin-converting enzyme inhibitors (ACEi), angiotensin II receptor blockers (ARB), loop diuretics, thiazide diuretics, potassium-sparing diuretics, non-steroidal anti-inflammatory drugs (NSAIDs), cancer chemotherapy and aminoglycosides. ${ }^{2,12,13,19,31}$ Corresponding ATC codes are detailed in the Supporting Information (Table S2). It was considered that the eligible drugs were chronically taken at home, according to the discharge letter and chronic conditions, even if they were not re-administered at hospital. Medication review was not performed routinely at the time of this study in the hospital. Consequently, drugs administered on the first day of hospitalization were also extracted if both experts deemed that the drug was taken at home. The experts were cautious in their judgment and did not include drugs in case of any doubt. Drugs administered after the improvement of AKI or drugs which are not usually prescribed in a community setting (e.g., intravenous aminoglycoside) were not included.

\section{Causality, Inappropriateness, And Avoidability}

The role of drugs in AKI events was assessed using the Naranjo scale (detailed in Table S4). ${ }^{32}$ This scale is a validated tool for evaluating the causal relationship between medication and the event. ${ }^{33}$ A score is attributed to each of ten axes concerning items such as the chronologic relationship between the drug and the event, or the de-challenge and re-challenge of the drug. The total score ranges from -4 to 13 and indicates the following probability categories: "unlikely" (score $\leq 0$ ), "possible" (score of 1 to 4), "probable" (score of 5 to 8), or "definite" (score $\geq 9$ ). We concluded that AKI was induced by a drug if the score category was "possible", "probable" or "definite". Drugs were evaluated independently from the patient's other medication. ${ }^{34}$ These criteria were applied at a single time point during the hospitalization corresponding to the admission. In this assessment, Cohen's Kappa was used to measure the extent of agreement between the two experts. The Kappa value was 0.72 , indicating good agreement (defined as a value between 0.6 and 0.80 ).

Inappropriateness of each prescription focused on medication overuse or misuse (dosage or duration), and was evaluated by using the "Screening Tool of Older People Prescriptions" (STOPP) criteria v.2. ${ }^{35}$ As STOPP criteria are based on diagnostic and/or prescription data, these criteria are explicit and do not require additional expert assessment.

Avoidability of CA-AKI events was estimated by using the Hallas criteria. ${ }^{36}$ The Hallas criteria are categorized into 4 classes as follows: "definitely avoidable": drug treatment was inconsistent with present-day knowledge; "possibly avoidable": prescription was not erroneous, but the event could have been avoided by an effort exceeding the obligatory demands; "not avoidable": the event could not have been avoided by any reasonable means, or was an unpredictable event in the course of a treatment fully with good medical practice; "unevaluable": data for which a rating could not be obtained or with conflicting evidence (Table S5). 
If the expert review found at least one drug with a Naranjo score $\geq 1$ and a precipitating factor, CA-AKI was considered as a multifactorial event.

\section{Statistical Analysis}

Statistical analyses and comparison of the two data tables provided by each reviewer were performed with $\mathrm{R}$ studio 1.0.143 software. Qualitative variables were reported as the number and percentage. Quantitative variables were reported as the mean and standard-deviation (SD), or (for non-normal distributions according to the Shapiro-Wilk test) as the median and interquartile range (IQR).

\section{Results}

\section{CA-AKI Events And Characteristics}

Over the two-year period, AKI events were screened among 4,767 inpatient stays of at least two days by patients aged 75 and over. After automated detection and double review, 713 CA-AKI were confirmed (15.0\%). Selection process is summarized in Figure 1. The mean age of patients corresponding to the 713 inpatient stays

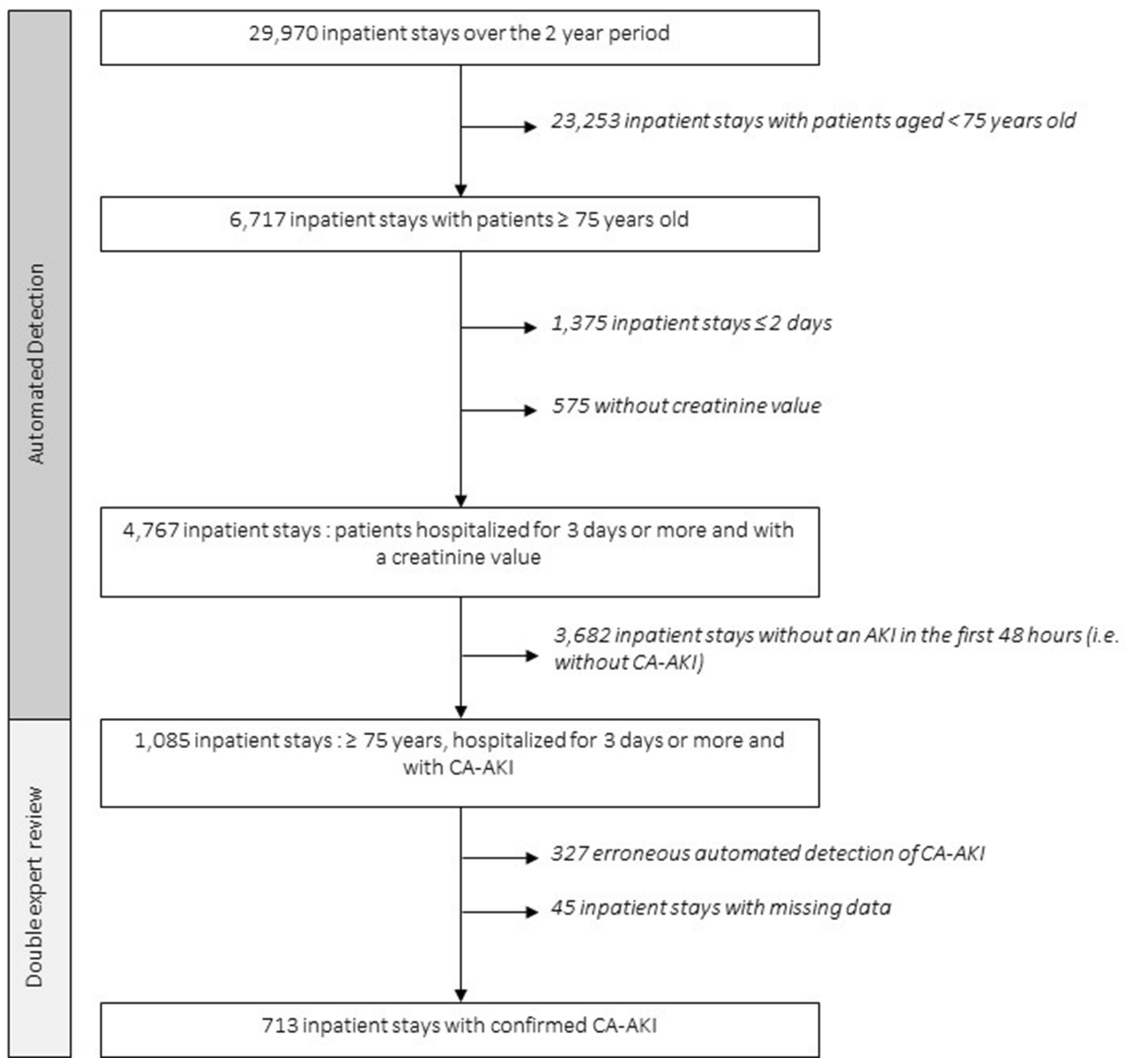

Figure I Diagram representing the inpatient stay selection process in 2 phases.

Abbreviations: AKI: acute kidney injury; CA-AKI: community-acquired acute kidney injury; KDIGO: kidney disease improving global guidelines. 
Table I Characteristics Of The 7I3 Confirmed CA-AKI Events

\begin{tabular}{|l|l|}
\hline Characteristics & $\begin{array}{l}\text { Inpatients Stays } \\
\text { (N=7 I3) }\end{array}$ \\
\hline Age, years (mean \pm sd) & $84.4 \pm 5.4$ \\
\hline Women, n (\%) & $504(70.7 \%)$ \\
\hline $\begin{array}{l}\text { Creatinine value at admission, mg/dL } \\
\text { (median \& IQR) }\end{array}$ & $1.4(1.0 ; 1.9)$ \\
\hline Stages of AKI according to KDIGO & \\
criteria, $\mathbf{n}$ (\%) & \\
Stage I & $585(82.0 \%)$ \\
Stage 2 & $10 \mathrm{I}(14.2 \%)$ \\
Stage 3 & $27(3.8 \%)$ \\
\hline Predisposing factors, $\mathbf{n}$ (\%) & \\
High blood pressure & $409(57.4 \%)$ \\
Diabetes & $173(24.3 \%)$ \\
Chronic heart failure & $1 \mathrm{II}(15.6 \%)$ \\
Chronic kidney disease & $64(9.0 \%)$ \\
\hline Precipitating factors, $\mathbf{n}$ (\%) & \\
Infection & $384(53.9 \%)$ \\
Dehydration & $147(20.6 \%)$ \\
Acute heart failure & $64(9.0 \%)$ \\
Rhabdomyolysis & $34(4.8 \%)$ \\
Surgery & $8(1.1 \%)$ \\
Ascites due to cirrhosis & $4(0.6 \%)$ \\
\hline
\end{tabular}

Abbreviations: sd: standard deviation; IQR: interquartile range; $\mathrm{AKI}$ : acute kidney injury.

was 84.4 (S.D. 5.4) and $70.7 \%$ of them were female. The characteristics of the CA-AKI events are described in Table 1 . AKI was mainly stage $1,(82.0 \%)$ according to KDIGO criteria. Infection was the most frequent precipitating factor and was identified in more than one in two cases. The experts identified 31 (4.3\%) obstructive AKI, which were secondarily excluded from the analysis of drug-induced events.

\section{CA-AKI Induced By Drugs}

The expert reviews rated 419 (58.8\%) AKI events as "possible", "probable" or "definite" ADRs according to the Naranjo scale. The expert review scored 276 AKI events as "possible", 139 as "probable" and 4 as "definite". Only one AKI event was scored as "unlikely" (i.e., a score of 0 ) and no drug known to be involved in CA-AKI was identified in 261 events (36.6\%). Results on Figure 2 show that a combination of two, three, four or even five drugs rated as "possible", "probable" or "definite" ADRs with the Naranjo scale was retrieved in $29.6 \%$ of AKI events.

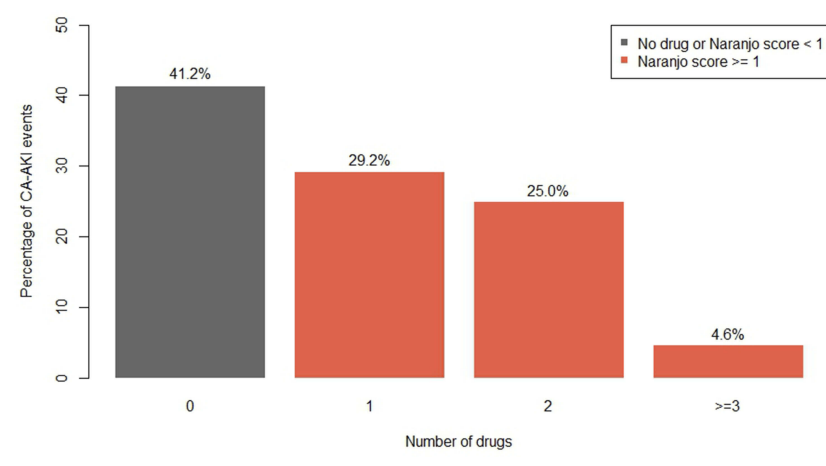

Figure 2 Distinction of the $7 / 3$ CA-AKI events according to the number of drugs with a Naranjo score $\geq$ I after double independent expert review.

\section{CA-AKI: A Multifactorial Event}

The analysis of the combination of precipitating factors and drugs revealed that $265 \mathrm{CA}-\mathrm{AKI}$ events were multifactorial, corresponding to $37.2 \%$ of all CA-AKI events and $63.2 \%$ of the drug-induced CA-AKI events. Results are presented in Table 2. A combination of one drug with a Naranjo score $\geq 1$ with precipitating factors was retrieved in 136 (19.1\%) AKI events. A combination of two drugs or more with a Naranjo score $\geq 1$ was identified in combination with precipitating factors in 129 (18.1\%) AKI events.

\section{Avoidability Of Drug-Induced CA-AKI Events}

Among all drug-induced CA-AKI and according to the Hallas criteria, the expert review deemed $280(66.8 \%)$ drug-induced CA-AKI as avoidable: $250(59.7 \%)$ AKI events as possibly avoidable and $30(7.1 \%)$ as definitely avoidable. The majority of the possibly avoidable druginduced CA-AKI were related to a required adaptation of drug therapy during intercurrent illness, mainly infection. For example, a combination of an ACEi/ARB with a diuretic, both with a Naranjo score $\geq 1$, was combined with infection in 95 (22.7\%) cases of drug-induced CA-AKI. Similarly, a combination of ACEi or ARB with a Naranjo score $\geq 1$ was combined with dehydration in $56(13.4 \%)$ cases of drug-induced CA-AKI. Both experts concluded that these drugs should have been reevaluated in this case.

\section{Inappropriateness Of Drugs Involved In CA-AKI Events}

Among the 666 drugs rated as "possible", "probable" or "definite" ADRs with the Naranjo scale, only 67 (10.0\%) drugs were found to be inappropriate according to the 
Table 2 Multifactorial CA-AKI Events Defined According To The Presence Or Absence Of Precipitating Factors And Drugs With A Naranjo Score $\geq 1$

\begin{tabular}{|c|c|c|c|c|c|}
\hline \multicolumn{3}{|c|}{ Independent Review By Two Experts } & \multirow[t]{2}{*}{ Interpretation } & \multirow[t]{2}{*}{ Conclusion } & \multirow{2}{*}{$\begin{array}{l}n(\%) \\
\left(n_{\text {total }}=713\right)\end{array}$} \\
\hline $\begin{array}{l}\text { Precipitating } \\
\text { Factors* }\end{array}$ & $\begin{array}{l}\text { A Single Drug With } \\
\text { A Naranjo Score } \geq I *\end{array}$ & $\begin{array}{l}\geq 2 \text { Drugs With A } \\
\text { Naranjo Score } \geq 1 *\end{array}$ & & & \\
\hline 0 & 0 & 0 & No drug nor precipitating factor & No cause & $87(12.2 \%)$ \\
\hline 1 & 0 & 0 & Precipitating factors alone & Unifactorial & $207(29 \%)$ \\
\hline 0 & 1 & 0 & A single drug with a Naranjo score $\geq 1$ & Unifactorial & $72(10.1 \%)$ \\
\hline 0 & 0 & 1 & $\geq 2$ drugs with a Naranjo score $\geq 1$ & Drug combination & $82(11.5 \%)$ \\
\hline I & I & 0 & $\begin{array}{l}\text { Precipitating factors AND a single } \\
\text { drug with a Naranjo score } \geq \text { I }\end{array}$ & Multifactorial & $136(19.2 \%)$ \\
\hline I & 0 & 1 & $\begin{array}{l}\text { Precipitating factors AND } \geq 2 \text { drugs } \\
\text { with a Naranjo score } \geq 1\end{array}$ & Multifactorial & $129(18.1 \%)$ \\
\hline
\end{tabular}

Notes: ${ }^{*} 0$ : absence of the criterion; I: presence of the criterion

STOPP criteria. ARB ( $\mathrm{n}=142 ; 21.3 \%)$ was one of the most frequent drug class involved in AKI events and was the most frequent, inappropriate prescription $(n=25 ; 17.6 \%$ of ARB prescriptions), according to the STOPP criteria. The main reason for this inappropriateness was the lack of indication. ACEi $(n=189 ; 28.3 \%)$ and loop diuretics $(\mathrm{n}=172 ; 25.7 \%)$ were also frequently involved in AKI events, but solely ACEi was rated as an inappropriate prescription ( $\mathrm{n}=19 ; 10.1 \%$ of ACEi prescriptions). Few prescriptions of potassium-sparing diuretics were retrieved by the expert review ( $\mathrm{n}=55 ; 8.2 \%)$, but they were frequently identified as inappropriate $(\mathrm{n}=19 ; 34.5 \%$ of potassium-sparing diuretic). Details can be seen in the Supporting Information (Table S3).

\section{Discussion}

\section{Main Findings}

Our study showed that drug involvement was frequent in CA-AKI and was found in $58.8 \%$ of cases. These druginduced CA-AKI covered $8.8 \%$ of hospital admissions in patients aged 75 and over, equal to around one in ten patients. Drug-induced CA-AKI was often multifactorial and around two thirds (63.2\%) could have been avoided by "an effort exceeding the obligatory demands". ${ }^{36}$ All these results highlight the importance of the prevention of druginduced CA-AKI, and that this prevention should take drug-disease interaction into account - especially in case of infection or dehydration.

\section{Comparison With Other Studies}

The incidence of CA-AKI varied between $0.7 \%$ and $4.3 \%$ according to studies. ${ }^{3,4,15,18}$ In our study, a CA-AKI was confirmed by an independent dual review in $15.0 \%$ of inpatient stays. This difference can first be explained by the age of inclusion at 75 and over in our study. AKI is a problem often found in older patients, and patients aged 70 or over are 3.5 times more likely to develop CA-AKI than younger patients. ${ }^{6}$ Age is, therefore, a risk factor for AKI. Thus, all patients in our study already ran the risk of developing AKI in addition to drugs and precipitating factors. Additionally, our method of detection and validation of CA-AKI included the appearance of AKI within the first $48 \mathrm{hrs}$ of hospitalization, and the improvement of AKI acquired at home prior to admission. Detection of CA-AKI was most often defined based on variation of the creatinine value within $48 \mathrm{hrs}$, with sometimes a diagnostic code (e.g., ICD-9 coding). ${ }^{2-4,16}$ The latter approach may under-estimate the real incidence of CA-AKI because there is an under-codification of AKI. ${ }^{14,16}$

\section{CA-AKI: A Multifactorial Event}

The involvement of drugs in CA-AKI was barely studied with a score of causality assessment. Wang et al reported a possible drug involvement in $59.9 \%$ of CA-AKI, based on a national cross-sectional study in China. ${ }^{18}$ Stucker et al found that $25.5 \%$ of CA-AKI had a combination of a diuretic and an $\mathrm{ACEi}$ or an $\mathrm{ARB} .^{2}$ In a retrospective study in Taiwan, $72.0 \%$ of patients with a CA-AKI used at least one drug with a nephrotoxic risk during the final 3 months leading up to the AKI, with a mean of 2.4 drugs per patient. ${ }^{16}$ Our study confirmed and emphasized these results. Based on an independent dual review, drug involvement was present in $58.8 \%$ of CA-AKI. This expert analysis of all confirmed cases permitted the identification of a multifactorial cause in $63.2 \%$ of drug-induced 
CA-AKI, because of the combination of a drug with a Naranjo score $\geq 1$ with at least one precipitating factor.

This result suggested a frequent combination of drugs and disease, consistent with the physiopathology of AKI and should be considered in CA-AKI prevention measures. A recent meta-analysis showed that continuing a treatment during an intercurrent illness increased the risk of developing an AKI by $15 \% .^{31}$ In our study, only $10.0 \%$ of drugs with a Naranjo score $\geq 1$ were inappropriate, according to STOPP criteria. Moreover, $66.8 \%$ of drug-induced CA-AKI were avoidable according to the Hallas criteria. Prevention based on potential inappropriate prescriptions lists seems to be inadequate for CA-AKI. However, this approach is frequently used to prevent drug-induced events in older patients, because it is easily implemented. ${ }^{37}$

\section{Prevention Of CA-AKI Events}

Therefore, it appears that new strategies are necessary for the prevention of CA-AKI. At first, a possible solution could be a systematic reevaluation of potentially nephrotoxic drugs during an intercurrent illness. A pilot study in the United Kingdom recommended a systematic cessation of medication during severe dehydration. Conclusions on the decrease of AKI were limited due to the absence of a control group; however, this intervention does not appear to pose a risk for patients. ${ }^{38}$ A second complementary approach could be the improvement of the safe use of drugs. Patient education on AKI at-risk situations is required and measures of prevention must be improved. In a 6-month prospective study conducted by Belaiche et al, $85 \%$ of patients with chronic kidney disease (CKD) were not aware of the CKD at-risk situations, particularly, the risk of AKI occurrence in the event of dehydration through ACEi or ARB treatments. ${ }^{39}$ In another study, $53 \%$ of community-based CKD patients reported that they were not aware of dehydration at-risk situations and $71 \%$ were not aware of what they should do in such situations. ${ }^{40}$

\section{Strengths Of The Study}

Some strengths can be found in our study: a large number of AKI events relative to other studies; the automated detection of CA-AKI events which were then validated by two independent experts; complementary skills and a dual review of all confirmed cases of CA-AKI; a computer interface (ADE-Scorecards ${ }^{\circledR}$ ) dedicated to the expert case review; a standardized approach, with the use of the STOPP criteria, the Naranjo scale, and the Hallas criteria; and a description of the combination of precipitating factors and drugs.

\section{Limitations Of The Study}

Our study had some limitations. The study was singlecenter study conducted in a single general hospital in northern France. Therefore, our results may reflect the prescribing habits of general practitioners operating within this area. Moreover, our study was limited to hospitalized patients aged 75 and over and did not include patients with CA-AKI who lived at home. Generalizations of our results must be made cautiously. Nevertheless, ADRs are most often observed in this age group, ${ }^{37,38}$ and the mean age of patients with CA-AKI events has also been high in studies which included patients as young as 18 years old. ${ }^{12-14,39}$ The threshold of $48 \mathrm{hrs}$ at admission was chosen so that we detected community-acquired AKI rather than hospital-acquired AKI. However, this cutoff might have included a number of genuine hospital-acquired AKI events having occurred during the first 2 days of hospitalization.

Our analysis of the medical records of the patients was retrospective, and we also studied communityacquired events. The lack of community data might have slightly incorrectly estimated the number of CAAKI. At the time of the study, medication reconciliation was not performed routinely in this hospital. Thus, some information may not have been recorded in the electronic health records, which may have been a potential source of error during the expert review. This potential lack of information may also under-estimate the number of obstructive AKI. Our retrospective approach may miss some CA-AKI: (i) in patients who did not have a creatinine measurement within the first 48 hrs; (ii) in patients who had only one creatinine measurement; (iii) in patients whose creatinine measurements were more than seven days apart. These three situations may mostly correspond to reassuring clinical situations because the basic biological parameters were not regularly monitored. The probability of AKI occurring in this context, therefore, seemed low, especially among hospitalized older patients.

Moreover, the period of study inclusion was 2012-2013 and some new drugs, such as sacubitril/valsartan, may have been identified in rare cases in a more recent period. However, 68 medications were associated with AKI in a recent study of pharmacovigilance database in France. ${ }^{12}$ Nearly all medications were prescribed 
during our study period, except bendamustine (2014). Our study did not identify contrast-induced CA-AKI because of the lack of reliable data concerning the administration of contrast agents in the days before hospital admission.

Items of the Naranjo scale were not all assessed in the study. Indeed, items 6 to 10 were scored "do not know" for most drugs because data were not most often available. This issue was raised by other authors. ${ }^{41,42}$

Another limitation was the use of the STOPP criteria, which may be less comprehensive than other tools for exploring the appropriateness of prescriptions (e.g., the Medication Appropriateness Index). However, STOPP criteria were chosen because they detect ADR at-risk situations and provide good reliability. ${ }^{35,36}$

The detection of CA-AKI did not include creatinine value that remained high (i.e., no improvement during inpatient stay). This mode of identification may pose a selection bias. Nevertheless, this bias was minimized as the hospital did not have an Intensive Care unit or Nephrology departments. Patients with severe AKI had to be admitted to another hospital.

Finally, this study was neither designed to identify synergistic and significant associations of risk factors (which can be determined through pharmacoepidemiological studies) nor was it designed for the description of AKI epidemiology. However, this study has usefully pathed the way for pharmacoepidemiological studies on a larger database with sufficient statistical power.

\section{Conclusion}

CA-AKI was a frequent problem in older patients at the time of hospital admission. These events were mostly multifactorial with an important drug involvement, and two-thirds seemed to be avoidable. The prevention of drug-induced CA-AKI could be carried out through a reevaluation of potentially nephrotoxic drugs (e.g., ACEi/ $\mathrm{ARB})$ during an intercurrent illness (e.g., infection).

\section{Acknowledgments}

We sincerely thank all the physicians who participated in the Patient Safety through Intelligent Procedure (PSIP) project in their respective departments, notably, Dr. Pascale Leurs, Dr. Olivier Brimont, Dr. Zine Baarir, and Dr. Philippe Lecocq. We thank Renaud Perichon and Sophie Quenton (health informatics engineers) for their invaluable assistance. We also thank Laurie Ferret and Joanna Dupont for preliminary work.

\section{Author Contributions}

All authors contributed to the conception, design of the study or acquisition of data, drafting or revising the article, gave final approval of the version to be published, and agree to be accountable for all aspects of the work.

\section{Funding}

This study was funded by the Fondation pour la Recherche Médicale (FRM). The FRM did not influence study design, analysis, data collection and presentation or interpretation of the results. The FRM did not review the manuscript prior to its publication.

\section{Disclosure}

Dr. Jean-Baptiste Beuscart reports grants from Fondation pour la Recherche Médicale, during the conduct of the study. The authors report no other conflicts of interest in this work.

\section{References}

1. Soto K, Campos P, Pinto I, et al. The risk of chronic kidney disease and mortality are increased after community-acquired acute kidney injury. Kidney Int. 2016;90(5):1090-1099. doi:10.1016/j.kint.2016.07.018

2. Stucker F, Ponte B, De la Fuente V, et al. Risk factors for communityacquired acute kidney injury in patients with and without chronic kidney injury and impact of its initial management on prognosis: a prospective observational study. BMC Nephrol. 2017;18:1. doi:10. 1186/s12882-017-0792-2

3. Hazara AM, Elgaali M, Naudeer S, Holding S, Bhandari S. The use of automated electronic alerts in studying short-term outcomes associated with community-acquired acute kidney injury. Nephron. 2017;135(3):181-188. doi:10.1159/000454779

4. Talabani B, Zouwail S, Pyart RD, Meran S, Riley SG, Phillips AO. Epidemiology and outcome of community-acquired acute kidney injury. Nephrology. 2014;19(5):282-287. doi:10.1111/nep.12221

5. Emmett L, Tollitt J, McCorkindale S, Sinha S, Poulikakos D. The evidence of acute kidney injury in the community and for primary care interventions. Nephron. 2017;136(3):202-210. doi:10.1159/000 460266

6. Rosner MH. Acute kidney injury in the elderly. Clin Geriatr Med. 2013;29(3):565-578. doi:10.1016/j.cger.2013.05.001

7. Forni LG, Dawes T, Sinclair H, et al. Identifying the patient at risk of acute kidney injury: a predictive scoring system for the development of acute kidney injury in acute medical patients. Nephron Clin Pract. 2013;123(3-4):143-150. doi:10.1159/000351509

8. Anderson S, Eldadah B, Halter JB, et al. Acute kidney injury in older adults. J Am Soc Nephrol. 2011;22(1):28-38. doi:10.1681/ASN.2010 090934

9. Coca SG. Acute kidney injury in elderly persons. Am J Kidney Dis Off J Natl Kidney Found. 2010;56(1):122-131. doi:10.1053/j.ajkd.20 09.12 .034

10. O'Connor MN, Gallagher P, Byrne S, O’Mahony D. Adverse drug reactions in older patients during hospitalisation: are they predictable? Age Ageing. 2012;41(6):771-776. doi:10.1093/ageing/afs046

11. De Figueiredo TP, De Souza G, Barroso SCC, do N, Reis AMM. Factors associated with adverse drug reactions in older inpatients in teaching hospital. Int J Clin Pharm. 2017;39(4):679-685. doi:10.10 07/s11096-017-0473-z 
12. Pierson-Marchandise M, Gras V, Moragny J, et al. The drugs that mostly frequently induce acute kidney injury: a case - noncase study of a pharmacovigilance database. Br J Clin Pharmacol. 2017;83 (6):1341-1349. doi:10.1111/bcp. 13216

13. Koza Y. Acute kidney injury: current concepts and new insights. J Inj Violence Res. 2016;8(1):58.

14. Schissler MM, Zaidi S, Kumar H, Deo D, Brier ME, McLeish KR. Characteristics and outcomes in community-acquired versus hospitalacquired acute kidney injury. Nephrology. 2013;18(3):183-187. doi:10.1111/nep. 12036

15. Wonnacott A, Meran S, Amphlett B, Talabani B, Phillips A. Epidemiology and outcomes in community-acquired versus hospital-acquired AKI. Clin J Am Soc Nephrol. 2014;9(6):1007-1014. doi:10.2215/CJN.07920713

16. Hsu C-N, Lee C-T, Su C-H, et al. Incidence, outcomes, and risk factors of community-acquired and hospital-acquired acute kidney injury: a retrospective cohort study. Medicine (Baltimore). 2016;95 (19):e3674. doi:10.1097/MD.0000000000003674

17. Mesropian PD, Othersen J, Mason D, Wang J, Asif A, Mathew RO. Community-acquired acute kidney injury: A challenge and opportunity for primary care in kidney health. Nephrol Carlton Vic. 2016;21 (9):729-735. doi:10.1111/nep.12751

18. Wang Y, Wang J, Su T, et al. Community-acquired acute kidney injury: a nationwide survey in China. Am J Kidney Dis. 2017;69 (5):647-657. doi:10.1053/j.ajkd.2016.10.034

19. Finlay S, Bray B, Lewington AJ, et al. Identification of risk factors associated with acute kidney injury in patients admitted to acute medical units. Clin Med. 2013;13(3):233-238. doi:10.7861/clinmedicine.13-3-233

20. Chang Y-P, Huang S-K, Tao P, Chien C-W. A population-based study on the association between acute renal failure (ARF) and the duration of polypharmacy. BMC Nephrol. 2012;13:96. doi:10.1186/1471-2369-13-96

21. Gong Y, Zhang F, Ding F, Gu Y. Elderly patients with acute kidney injury (AKI): clinical features and risk factors for mortality. Arch Gerontol Geriatr. 2012;54(2):e47-e51. doi:10.1016/j.archger.2011.05.011

22. Loboz KK, Shenfield GM. Drug combinations and impaired renal function-the 'triple whammy.'. Br J Clin Pharmacol. 2005;59 (2):239-243. doi:10.1111/j.1365-2125.2004.02188.x

23. WHO International Classification of Diseases. WHO International Classification of Diseases. Available from: http://www.who.int/classi fications/icd/en/. Accessed October 21, 2019.

24. WHO The Anatomical Therapeutic Chemical Classification System with Defined Daily Doses (ATC/DDD). WHO The Anatomical Therapeutic Chemical Classification System with Defined Daily Doses (ATC/DDD). Available from: http://www.who.int/classifica tions/atcddd/en/. Accessed March 6, 2018.

25. IUPAC International Union of Pure and Applied Chemistry. International Union of Pure and Applied Chemistry. Available from: https://iupac.org/. Accessed March 6, 2018.

26. Chazard E, Mouret C, Ficheur G, Schaffar A, Beuscart J-B, Beuscart R. Proposal and evaluation of FASDIM, a fast and simple de-identification method for unstructured free-text clinical records. Int $\mathrm{J}$ Med Inf. 2014;83(4):303-312. doi:10.1016/j.ijmedinf.2013.11.005

27. Chazard E, Băceanu A, Ferret L, Ficheur G. The ADE scorecards: a tool for adverse drug event detection in electronic health records. Stud Health Technol Inf. 2011;166:169-179.
28. Beuscart R, Hackl W, Nøhr C. Detection and prevention of adverse drug events - information technologies and human factors. Preface Stud Health Technol Inform. 2009;148:v-vi.

29. Levey AS, Stevens LA, Schmid CH, et al. A new equation to estimate glomerular filtration rate. Ann Intern Med. 2009;150 (9):604-612. doi:10.7326/0003-4819-150-9-200905050-00006

30. Matsushita K, Mahmoodi BK, Woodward M. Comparison of risk prediction using the CKD-EPI equation and the MDRD study equation for estimated glomerular filtration rate. JAMA. 2012. doi:10. 1001/jama.2012.3954

31. Whiting P, Morden A, Tomlinson LA, et al. What are the risks and benefits of temporarily discontinuing medications to prevent acute kidney injury? A systematic review and meta-analysis. BMJ Open. 2017;7(4):e012674. doi:10.1136/bmjopen-2016-012674

32. Naranjo CA, Busto U, Sellers EM, et al. A method for estimating the probability of adverse drug reactions. Clin Pharmacol Ther. 1981;30 (2):239-245. doi:10.1038/clpt.1981.154

33. Khan LM, Al-Harthi SE, Osman A-M-M, Sattar MAAA, Ali AS. Dilemmas of the causality assessment tools in the diagnosis of adverse drug reactions. Saudi Pharm J SPJ Off Publ Saudi Pharm Soc. 2016;24(4):485-493. doi:10.1016/j.jsps.2015.01.010

34. Agbabiaka TB, Savović J, Ernst E. Methods for causality assessment of adverse drug reactions: a systematic review. Drug Saf. 2008;31 (1):21-37. doi:10.2165/00002018-200831010-00003

35. O’Mahony D, O’Sullivan D, Byrne S, O'Connor MN, Ryan C, Gallagher P. STOPP/START criteria for potentially inappropriate prescribing in older people: version 2. Age Ageing. 2014;44(2):213218. doi:10.1093/ageing/afu145

36. Hallas J, Harvald B, Gram LF, et al. Drug related hospital admissions: the role of definitions and intensity of data collection, and the possibility of prevention. $J$ Intern Med. 1990;228(2):83-90. doi:10.1111/j.1365-2796.1990.tb00199.x

37. Lavan AH, Gallagher P. Predicting risk of adverse drug reactions in older adults. Ther Adv Drug Saf. 2016;7(1):11-22. doi:10.1177/204 2098615615472

38. Morrison C, Wilson DM MEDICINE SICK DAY RULES CARDS UPDATE TO EVALUATION.:3.

39. Belaiche S, Romanet T, Allenet B, Calop J, Zaoui P. Identification of drug-related problems in ambulatory chronic kidney disease patients: a 6-month prospective study. J Nephrol. 2012;25(5):782-788. doi:10. 5301/jn.5000063

40. Belaiche S, Mercier E, Cuny D, et al. Community pharmacists' interventions to prevent and screen chronic kidney disease patients Nephrol Ther. 2017;13(2):87-92. doi:10.1016/j.nephro.2016.06.006

41. Shalayel MH, Mohamed Ayed IA, Huneif MA, Mohammed Kordofani Y. A retrospective evaluation of cutaneous adverse drug reactions (CADRs) due to antibiotics using naranjo adverse drug reactions (ADRs) probability scale. J Young Pharm. 2018;10 (1):113-116. doi:10.5530/jyp.2018.10.25

42. Mittal N, Gupta MC. Comparison of agreement and rational uses of the WHO and Naranjo adverse event causality assessment tools. $J$ Pharmacol Pharmacother. 2015;6(2):91-93. doi:10.4103/0976-50 0X.155486
Clinical Interventions in Aging

\section{Publish your work in this journal}

Clinical Interventions in Aging is an international, peer-reviewed journal focusing on evidence-based reports on the value or lack thereof of treatments intended to prevent or delay the onset of maladaptive correlates of aging in human beings. This journal is indexed on PubMed Central, MedLine, CAS, Scopus and the Elsevier
Bibliographic databases. The manuscript management system is completely online and includes a very quick and fair peer-review system, which is all easy to use. Visit http://www.dovepress.com/ testimonials.php to read real quotes from published authors. 